Association for Information Systems AIS Electronic Library (AISeL)

AMCIS 2002 Proceedings

Americas Conference on Information Systems

(AMCIS)

December 2002

\title{
GLOBAL SERVICE PROVIDER STRATEGIES AND NETWORKING ALTERNATIVES
}

Mahesh Raisinghani

University of Dallas

Follow this and additional works at: http://aisel.aisnet.org/amcis2002

\section{Recommended Citation}

Raisinghani, Mahesh, "GLOBAL SERVICE PROVIDER STRATEGIES AND NETWORKING ALTERNATIVES" (2002). AMCIS 2002 Proceedings. 139.

http://aisel.aisnet.org/amcis2002/139

This material is brought to you by the Americas Conference on Information Systems (AMCIS) at AIS Electronic Library (AISeL). It has been accepted for inclusion in AMCIS 2002 Proceedings by an authorized administrator of AIS Electronic Library (AISeL). For more information, please contact elibrary@aisnet.org. 


\title{
Global Service Provider Strategies AND NETWORKING ALTERNATIVES
}

\author{
Mahesh Raisinghani \\ University of Dallas \\ mraising@gsm.udallas.edu
}

\begin{abstract}
This paper focuses on network planning strategies for the global communications carrier. It discusses strategic planning for the provision of new data and Internet services, and assesses alternative network designs and technologies to provide optimized solutions and service delivery. The discussion covers the regional, metropolitan and local, or last mile, networks and related access technologies. The main objectives of this paper include development of a framework and methodology for network modeling and analysis to support international business development with strategies for metropolitan and local access networks; determination of the network cost drivers for providing voice and IP services to various market segments, including the small $\&$ medium enterprise (SME) and multi-dwelling unit (MDU) markets; identification of the market and cost synergies for combining voice and IP services on common carrier facilities; and comparison of leasing versus building alternatives for metropolitan transport and last mile access networks.
\end{abstract}

Keywords: Network planning strategies, global communications carrier, network architectures, strategic implementations, future network considerations

\section{Introduction}

This paper focuses on network planning strategies for the global communications carrier. It discusses strategic planning for the provision of new data and Internet services, and assesses alternative network designs and technologies to provide optimized solutions and service delivery. The discussion covers the regional, metropolitan and local, or last mile, networks and related access technologies. Telecommunications is changing rapidly and extending beyond regional and national country boundaries into the global arena. The boundaries that have previously defined the telecom competitive landscape in the recent past, such as IXC vs. LEC vs. CLEC, are blurring in a market being defined by mergers, acquisitions and joint ventures (Martin, Deskey, and Pihl, 1999). At the same time, there is a growing trend away from traditional voice services to data-centric IP-based services. The communications customer is also becoming more sophisticated with new technical and service requirements, while the traditional markets and segments are fast changing. Telecommunications end users are increasingly savvy, gaining insight into provider capabilities and raising their expectations of provider performance as a result (Martin et al, 1999). Customer satisfaction and loyalty depend more than ever on the carrier's ability to understand and meet these changing requirements.

A framework and methodology to assess and analyze the telecommunications networking requirements and alternatives to the global carrier becomes very important. A framework and methodology helps the carrier identify and understand regional characteristics of a given country. It also helps in assessing city types, which may vary by functionality and market potential. The carrier needs to consider other key attributes such as: demand for voice and data services; volume of traffic staying and leaving varied parts of the country network; geometry of network elements and facilities; and competition. As the global carrier considers entry into new markets, its strategic planning should include: analysis of target countries; assessment of regional transport networks connecting targeted cities; and assessments of the metropolitan (metro) transport and local loop network requirements within targeted cities. These requirements will be driven by the local market conditions, and the carrier's requirements for network build and deployment.

The main objectives of this paper include: 
- Development of a framework and methodology for network modeling and analysis to support international business development with strategies for metropolitan and local access networks;

- Determination of the network cost drivers for providing voice and IP services to various market segments, including the small $\&$ medium enterprise (SME) and multi-dwelling unit (MDU) markets;

- Identification of the market and cost synergies for combining voice and IP services on common carrier facilities; and

- Comparison of leasing verses building alternatives for metropolitan transport and last mile access networks.

The discussion opens with a description of the general problem and then identifies and discusses key drivers and factors, trade-offs and alternatives that are faced by the global communications carrier. The discussion closes with some general recommendations and a summary of conclusions.

\section{Discussion of European Business Strategies for Serving Local/Metro Markets}

\section{Business Opportunities}

The strategic planning for a global communications carrier involves the interplay of business, marketing, technology, and regulatory factors. While some of these factors are independent of one another, most are inter-dependent on each other. One example of an inter-dependent factor is a country's policy regarding wireless spectrum - this may influence the technology choices, network costs and services offered. Figure 1 shows some of the dynamic factors of network planning that the global communications carrier may consider. Carriers entering new markets must deal with these dynamics. They must determine how to take advantage of technology, the changing regulatory environment, existing competitive service providers, and new customer demands -- to build optimal networks and successful businesses (Wrede, 2000). The choice of network technology is more important than ever due to today's rapidly changing market. One of the factors considered in the general discussion include business opportunities. The carrier may choose to offer IP only wholesale, PTT by-pass or a full suite of telecom services. Another factor centers on target market segment decisions, such as residential, SME or large enterprises. A third decision is network infrastructure ownership, such as end to end ownership of the network, leasing, or partnering with other entities. And a fourth issue regards positioning decisions to specific market segments - such as IP only services to SME and large enterprises, or wholesale IP dial-up access to residential focused Internet service providers (ISPs).

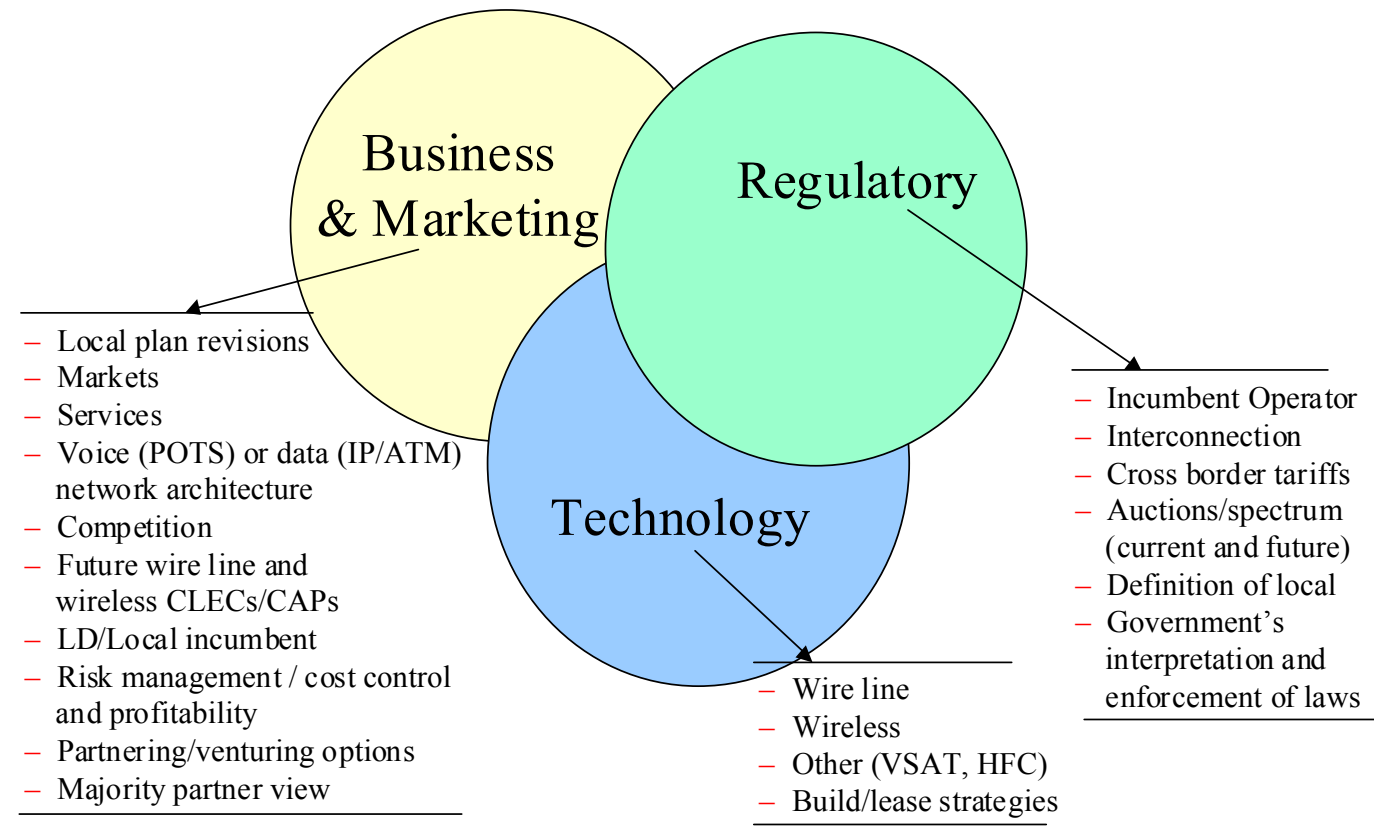

Figure 1. Dynamics of Network Planning 


\section{Target Market Segment and Positioning Decisions}

The global communications carrier must clearly define its strategy with respect to market segments, customers served, and services and applications delivered. This must include a view of today's market demand, including competition, and a view of the near-term future. The market requirements will be an integral component to meeting carrier objectives, and will drive its decisions relating to technologies and network architectures. The carrier must consider the following aspects in its marketing decisions:

- $\quad$ Market segment(s) served;

- Positioning decisions (i.e. wholesale, retail, full service) to specific market segments;

- $\quad$ Market demand;

- Customer requirements;

- Competitive factors; and

- Specific services and applications provided.

Many of the above factors have a current "today" element and a future element (i.e. a few years out). These marketing decisions and overall plans drive technology choices that best deliver the services and applications demanded. They also drive network architecture choices regarding scalability (to grow), flexibility (to meet changing needs), and how best to leverage available access technologies. Large service providers need to focus on market opportunities that leverage their core strengths, including technology and networks, while at the same time recognizing that there are smaller carriers pursuing niche markets with differentiated capabilities (Strouse, 2001). Different market segments will require different services and varying amounts of access bandwidth or capacity. These different market segments will be served by alternative technologies that minimize overall costs. Figure 2 illustrates sample market segments and their respective bandwidth, services and technologies. Most large enterprises will require a high amount of bandwidth (i.e. multiple E-3s or STM-1s) that support voice and data private lines, and virtual enterprise networking. Fiber optic connections built directly into a physical serving location (i.e. customer building) may be an optimal solution. SMEs that require less bandwidth may be better served with other alternative access technologies, including fixed wireless access (FWA), digital subscriber line (DSL), and existing copper facilities.

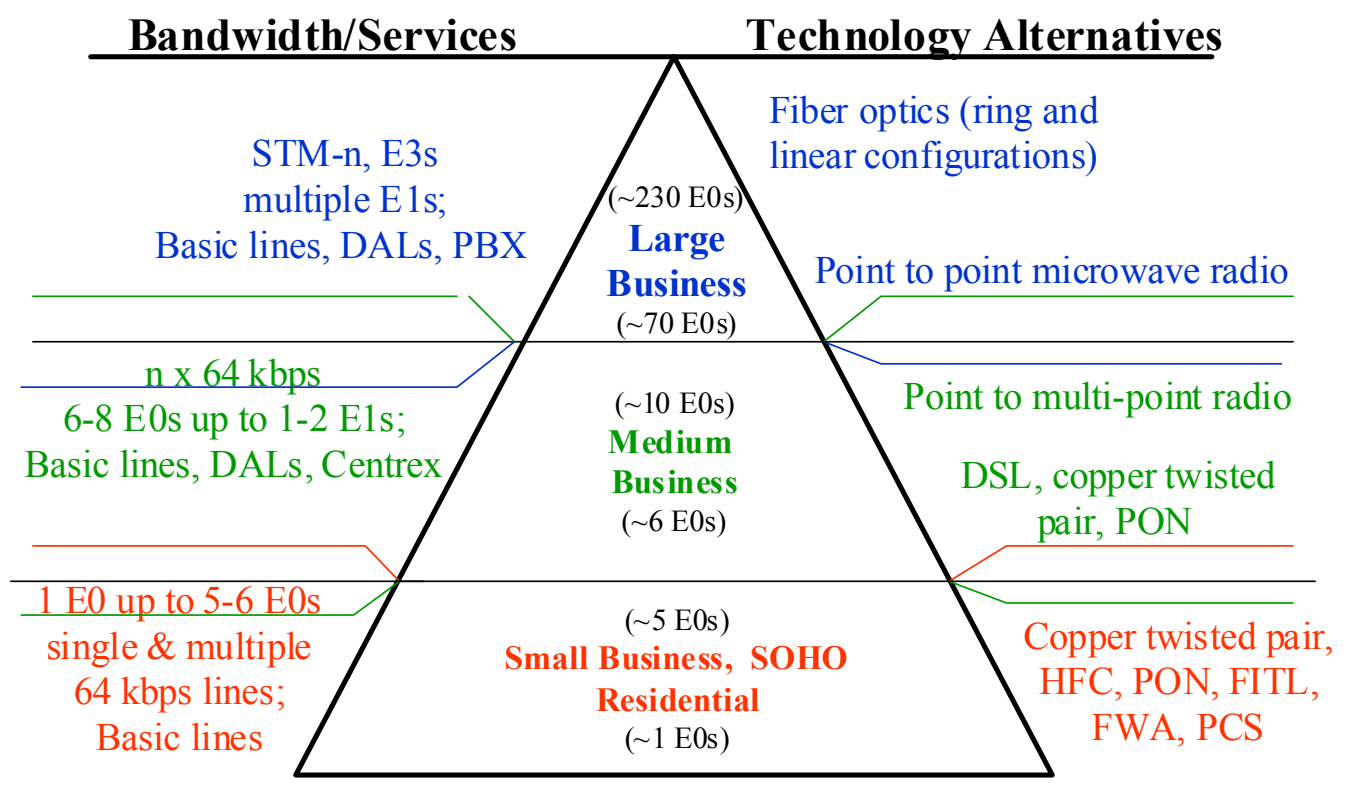

Figure 2. Market Segmentations

\section{Network Infrastructure Ownership Decisions}

Network infrastructure decisions relate to the different aspects of the global carrier's network. The network can be divided into four segments. The first segment is the core or backbone network, which includes plant and facilities that span across a country 
or countries. This part of the network typically uses high-speed optical transport technologies to carry bits of information across national boundaries. The second segment of the network is the regional network. This is a sub-network of the core network interconnecting cities within a geographic region or area of a country. This network also uses high-speed optical transport technologies to carry traffic between cities. The third segment covers the network within a city -- connecting various switching, routing and traffic aggregation facilities across a metropolitan or city area. The final network segment comprises the access network or last mile. This is the portion of the network that connects the customer sites to the carrier's network facilities. Figure 3 shows a conceptual city network - focusing on the metropolitan (city) and access (last mile) networks. This network may be comprised of various metropolitan and collector fiber optic rings, in addition to: point to point fiber connections, radio or wireless access, and leased facilities. The global carrier must consider various network infrastructure ownership decisions. Some of the key decisions include:

- Leasing or buying plant and facilities - this decision impacts costs, control of infrastructure, and time to market. It is more likely that a carrier will build core and regional infrastructure, and lease the metro/access plant and facilities; and

- Leasing last mile tariffed facilities from incumbent carriers vs. deploying alternative technologies such as FWA or DSL to connect to the customers. Costs, regulations regarding the unbundling of incumbent carrier networks, and the competitive environment influence this decision.

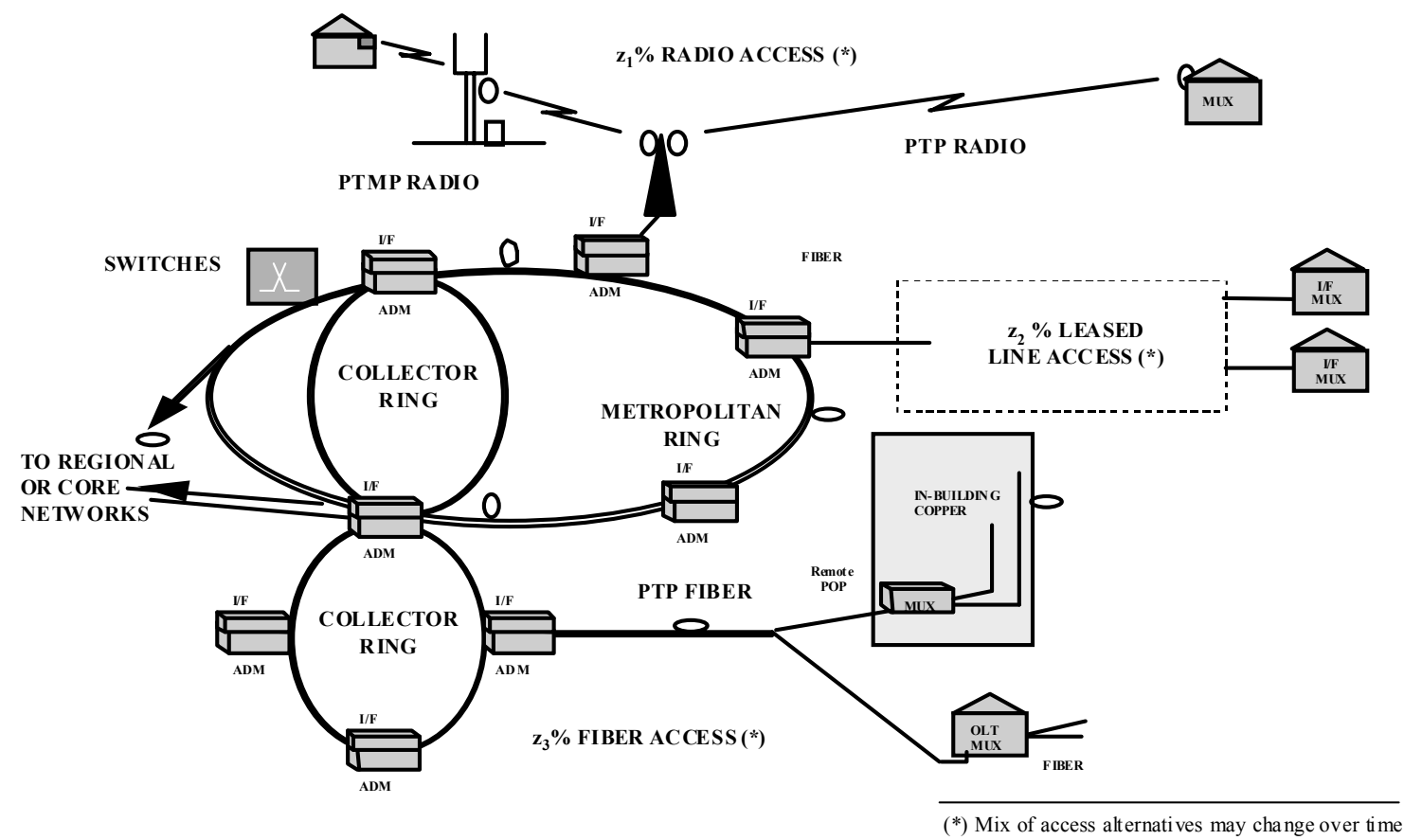

Figure 3. Conceptual City Network

As the global carrier weighs these decisions, the business, market positioning, regulatory and technology dynamics come into play. Albright (2001) talks about the impact of disruptive technologies to the carrier. Global carriers are going through a significant transition in their networks and associated technologies. They must identify disruptive technologies at the right time during this transition -- taking advantage of innovations by turning them into strategic opportunities. Albright describes these new technologies as having the following characteristics: revolutionize cost structures; increase network capacity; enhance quality of service; improve performance; but notes that they are difficult to incorporate into legacy carrier networks and systems. Wrede (2000) further explains that successful service providers will need to understand the economics of delivering advanced services to their customers and how technology will impact those economics. 


\section{Considerations of Generic Models}

\section{Services, Market Segments, Network Architectures and Cost Drivers}

This section continues to develop the general problem and challenges faced by the global communications carrier. There are different network architectures that provide varied levels of service to the customer. Each architecture varies in complexity, network functionality, services supported and overall network costs. As the carrier defines its business objectives, it can then determine an appropriate market strategy. The carrier objectives and market strategies drive the selection of the architectures and technologies that best meet these plans. The network architectures and technologies will vary by cost, risk, flexibility, scalability, complexity and time to market considerations.

\section{Low Capital Intensity - IP Only}

The first model, "low capital intensity - IP only" network model, provides the carrier with an option to sell public Internet access and IP virtual private networking services with minimal infrastructure build-out. The IP-only network requires the lowest outlay of capital investment - a minimal set of services would require only network router/server facilities in the metropolitan and regional networks, and associated back office systems. Refer to Figure 4 and note the required network infrastructure, denoted in yellow -- the rest of the infrastructure being leased. The carrier could increase the amount of capital investment by varying how much of the network is leased, owned or partnered with other entities. This network could serve large enterprise, SME and residential markets - in addition to wholesaling capacity to residential focused ISPs.

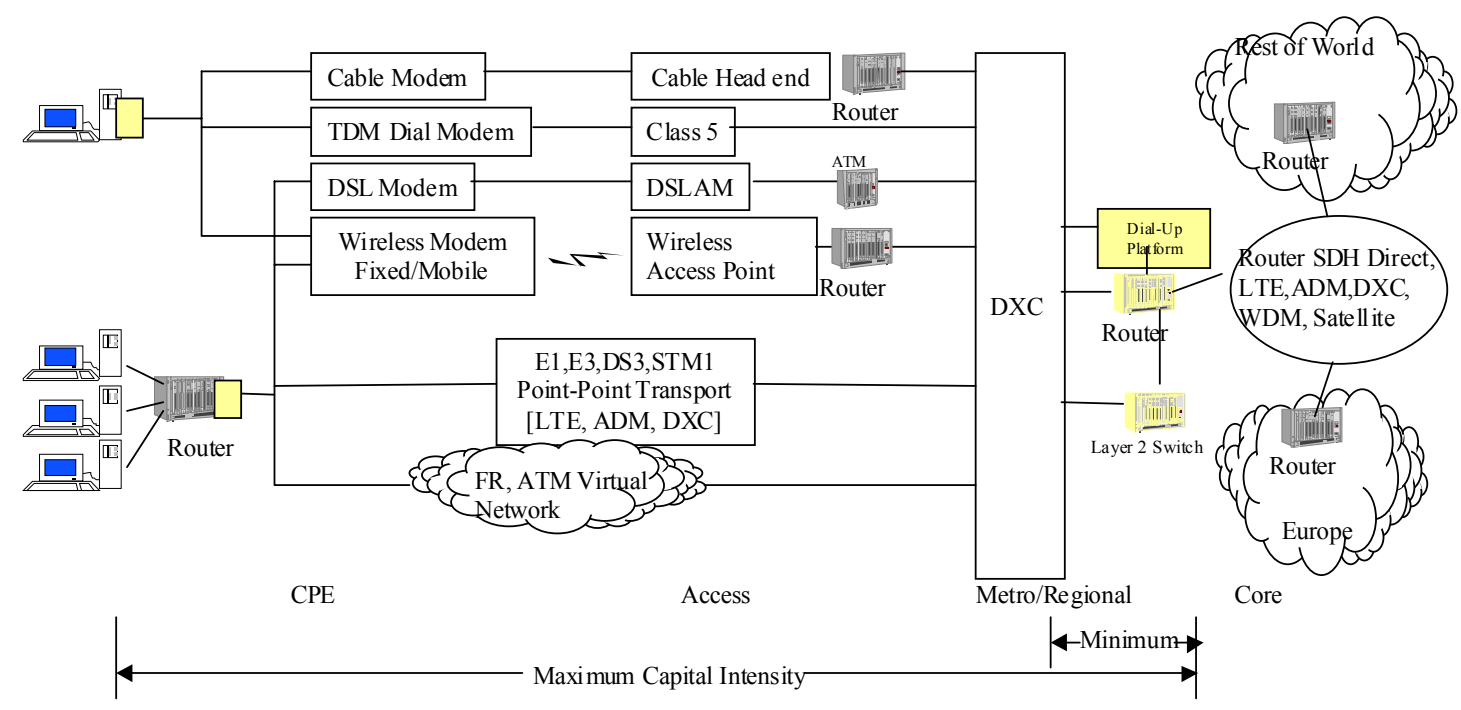

Figure 4. Low Capital Intensity Network

\section{Medium Capital Intensity - IP + International Voice}

The second model, "medium capital intensity - IP + international voice" network model, expands on the first model. This network adds voice over Internet (VoIP) and competitive PTT services, such as: local by-pass, toll and international switched voice, private line and data services. As the IP services grow, the carrier needs to introduce private line and digital cross connect (DXC) capabilities into the network to more efficiently groom the access traffic. This network also includes long distance (LD) voice switches. Frame relay and ATM may also be needed to serve as data link layer technologies for the transport of IP services. This network infrastructure will support frame relay and ATM data services, private line services, and connectivity to international PSTN dial services. Refer to Figure 5 and note additional infrastructure, which is denoted in green -- again the remainder being leased. As infrastructure is added, the capital investment, ownership and control of the network increases for the carrier. 


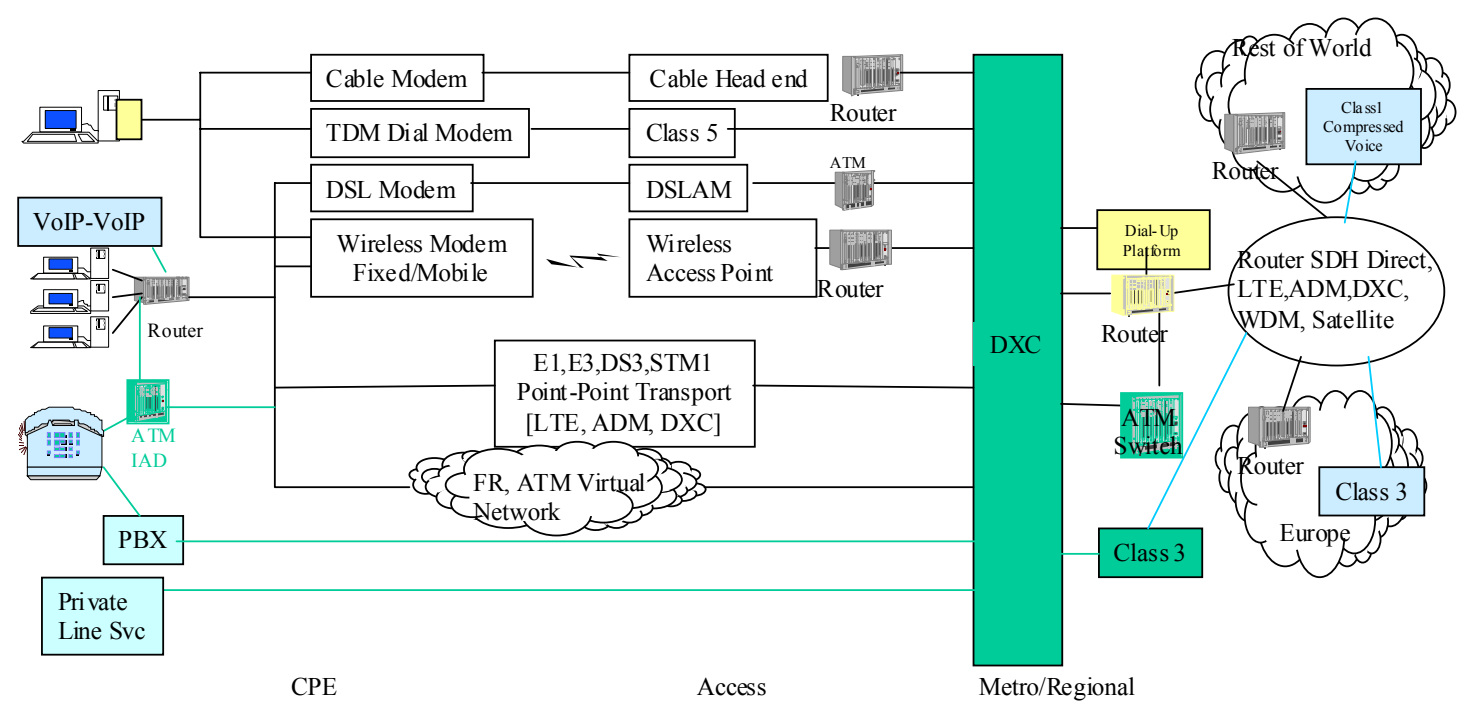

Figure 5. Medium Capital Intensity Network

\section{High Capital Intensity - IP + International Voice + Select Local PSTN Voice}

The third model, "high capital intensity - IP + international voice + select local PSTN voice" network model, expands on the first and second models. This network adds IP-only services such as: VoIP to PSTN, web hosting, security, mirroring of sites, and alternative service provider (ASP) services. It also provides switched local voice to selected market segments (i.e. SME, large enterprise) and international voice to residential customers. The network requires VoIP to PSTN gateways, intelligent customer equipment, and local/LD voice switches. Refer to Figure 6 and note the additional infrastructure, denoted in magenta -- the remainder being leased. This network targets local services to support Pan-European and international customers.

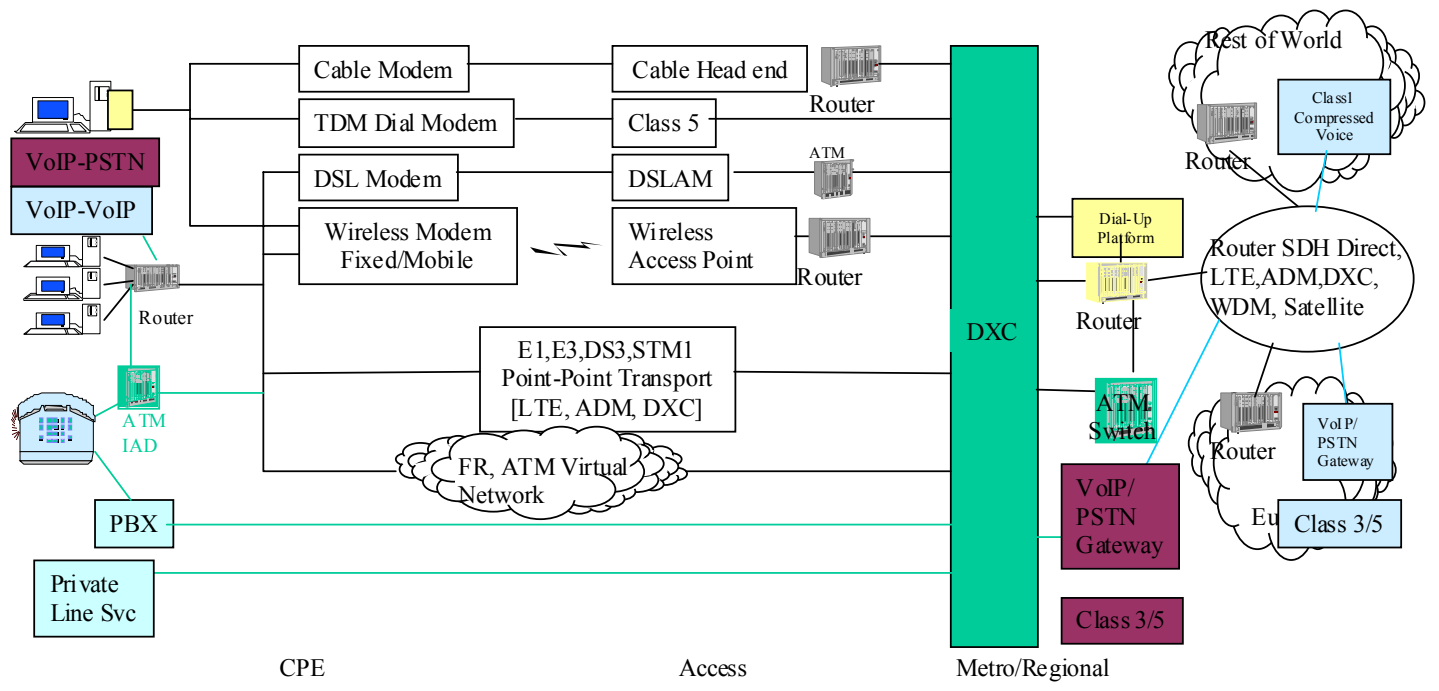

Figure 6. High Capital Intensity Network 


\section{Very High Capital Intensity - Full Suite of Local to Global Services}

The final model, "very high capital intensity - full suite of local to global services" network model, expands on the previous three models. This network adds VoIP, switched local voice, international voice, private line and data services to the residential market segment. This network requires local voice switches deployed in the metro/local networks. Refer to Figure 7, noting additional infrastructure, denoted in red -- the remainder being leased.

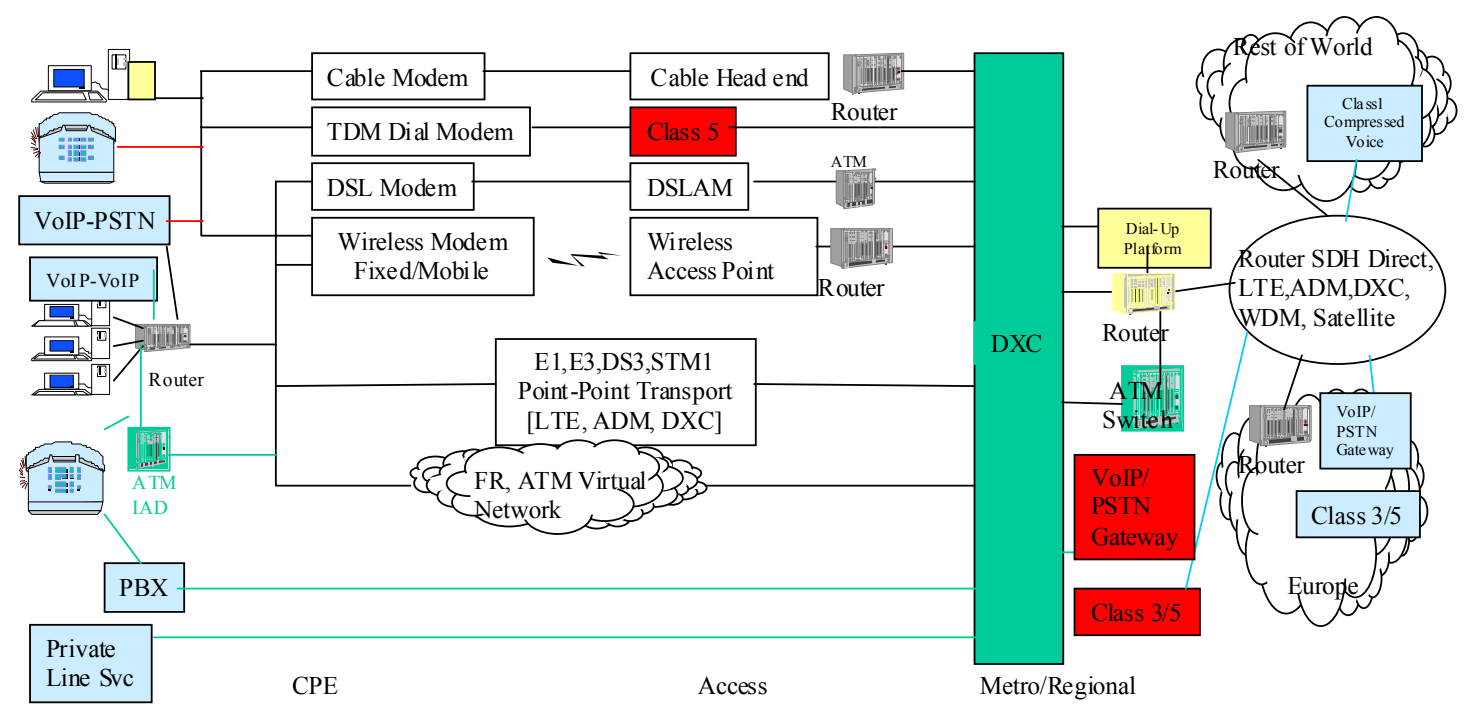

Figure 7. Very High Capital Intensity Network

This network has the highest outlay of capital investment, but supports a full suite of local to global services. This network architecture focuses on the entire local market, including the residential market segment.

\section{Network Design}

A network design process example is presented in Figure 8. A network planning team could use this process to facilitate their decisions. The steps in this process include the following:

- Establish unconstrained design goals (i.e. lowest capital outlay, scalability, flexibility to offer array of services, performance);

- Perform network modeling and analysis of backbone, regional, metro and access networks, requiring the following inputs:

- Equipment architecture options,

- Network topology,

- Traffic demand data,

- Design parameters,

- Equipment cost parameters,

- Network selection criteria;

- Perform "internal" carrier designs of the network using software tools (i.e. simulations);

- Coordinate "external" vendors network designs, including equipment costs and counts;

- Compare "internal" and "external" network designs, and based upon selection criteria, select best architectures and technologies;

- Identify prioritized requirements based upon carrier's resource constraints;

- Assess selected architectures and technologies based upon cost and performance trade-offs;

- If selected architectures and technologies do not meet cost and performance criteria, then repeat network modeling and analysis with revised assumptions and inputs; 
- Selected architectures and technologies meeting cost and performance trade-offs would yield viable alternatives. These alternatives would provide estimates of the following over a multi-year period:

- Network evolution plan,

- Network capital outlays (costs),

- Power and space requirements for network facilities (i.e. switch/router sites),

- Costs of back-hauling traffic verses switching traffic locally within a city or metro area, and

- Network operational costs, including systems and information technology (IT) costs.

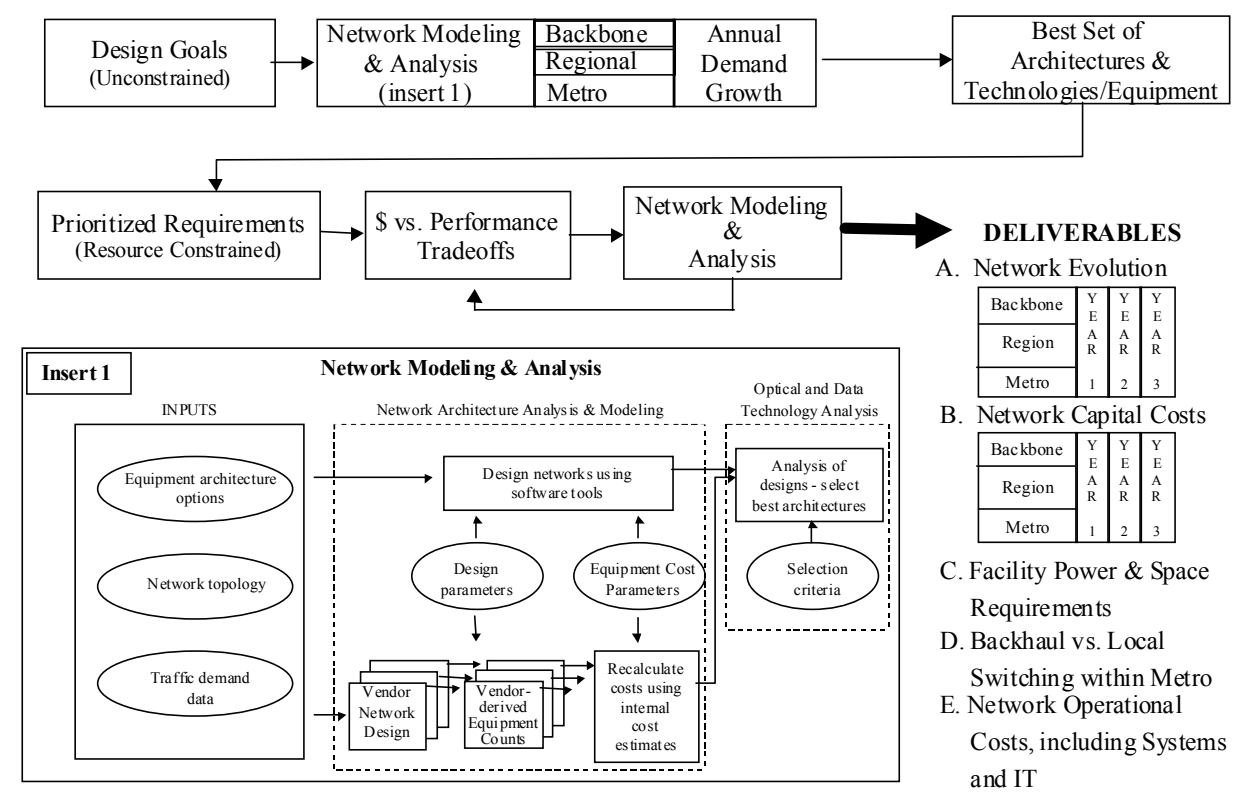

Figure 8. Network Design Process

\section{Strategic Implementations Considered}

There are a variety of strategic options that can be considered for the global communications carrier. Among these options include: all leased facilities, including last mile access, metro and regional transport; leased last mile and built metro and regional transport - including outside plant construction of fiber plant, facilities, network electronics and equipment; leased last mile, leased dark fiber for metro and regional transport, and built facilities, electronics and equipment; and deployed DSL for last mile access, leased dark fiber for metro and regional transport, and built facilities, electronics and equipment. Other variations, not considered, included: using wireless access - a combination of point to multi-point and mobile - for last mile access; or securing and upgrading cable plant (coaxial cable) facilities for bi-directional voice and high speed data. Each of these alternatives has their respective strategic benefits and drawbacks to the global communications carrier. Among the factors to be weighed and considered by the carrier include:

- Level of risk assumed by the carrier;

- Ownership and control of infrastructure;

- Control of customers and services provided;

- Flexibility to add capacity and meet changing customer technical requirements;

- Time-to-market - a wireless access with leased transport facilities alternative may allow the carrier quicker entry into a given market;

- Ability to scale or grow the network as customers are added - it may be hard to secure additional leased facilities or higher capacity facilities from an incumbent competitive carrier;

- Trade-offs of capital outlays verses operating costs; and

- Timing of investment - building network infrastructure requires large up-front capital outlays, while leasing results in recurring expenditures tied more closely to adding customers. 


\section{Recommendations for Italian Regional Network}

The potential for significant regional, metro and access network cost synergies between the "ISP network" and the "traditional voice and data network" could be realized if the carrier did the following:

- Target a common set of customers (i.e. SME);

- Deliver bundled voice and data services to customers;

- Optimize the network for specific market segments (i.e. SME);

- Leverage unbundled local loops with DSL or other combinations of economical broadband access technologies for last mile access;

- Focus on islands of dense customer coverage in given geographic markets; and

- Establish on-net or direct connections via leased dark fiber or building fiber infrastructure in the metro and regional transport networks.

\section{Other Future Network Considerations}

The global communications carrier might consider additional aspects of network design and planning. One aspect is distributed switching and moving network intelligence closer to the customer to reduce costs and provide network flexibility. A second aspect is packet technologies and packet-based network architectures to reduce costs, and improve access and transport efficiencies. And a final aspect is optical capabilities to reduce costs, increase efficiencies, and improve service delivery in the regional and metro networks.

\section{Distributed Switching and Pushing Intelligence to Network Edge}

any vendors and service providers are working together to develop next generation switch architectures. These new switches will distribute processing functions and intelligence out into the network closer to the customer. The distributed switch architecture promises to: lower carrier costs; support dominant data and IP network traffic; provide deployment flexibility; extend control and capabilities to the customer to manage their services; and provide intelligence to allow the network to manage services. A big driver of the next generation switch is the ability for carriers to provide new services - faster and easier. The next generation switch provides:

- Distributed network architecture;

- Logical connection of gateways, controllers, broadband core and services;

- Best-of-breed components based on industry standard protocols; and

- $\quad$ Supports IP and ATM core technologies.

The traditional switch uses a centralized architecture that provides ports, switch matrix, call control and feature processing in a common physical platform. The distributed switch architecture separates these components. It distributes the ports and switch matrix throughout the network, to the edge where the customer resides. It also separates the control and feature server functions for added flexibility. It takes advantage of various gateway devices that connect and provide intelligence across the network. According to Katz (2000), the carrier move from PSTN to the next generation packet network is in its third stage of development. The first stage was the build out of optical networks for efficient transport. The second stage was the development and deployment of soft-switch (next generation switch) technologies necessary to direct call traffic on the new networks. This stage also included the development of new protocols and standards, to which basic features could be supported across the network. The third stage focuses on the development of enhanced services and feature sets.

\section{Packet Technologies and Packet-based Architectures}

Another area of recent interest by carriers has been in packet aggregation and switching capabilities, leveraging LAN-based technologies. Carriers are interested in packet-based technologies for the following reasons:

- There is a new LAN paradigm - today's LAN technology has evolved to support high speed full duplex gigabit Ethernet (GbE) packet networks; 
- There is significant forecasted growth of data and IP traffic - Ethernet is a de-facto native protocol for IP and most traffic data originates as Ethernet frames; packet technologies could prove efficient for handling this future Ethernet traffic;

- There are key enablers of native data technologies - such as the availability of high bandwidth resources (i.e. fiber) and wavelength division multiplexing (WDM) technologies;

- New service and cost models are driving carriers to look at packet-based technologies; and

- There are major potential benefits, including: lower costs to customers and carriers; enabler of new services and revenue sources; ability to support existing carrier services; easier provisioning and service delivery; and lower operational costs (i.e. network maintenance labor).

Packet aggregation and switching technologies become important as customer profiles change to more data-centric services and applications. Services can be provided over a single access facility and switch/router interface resulting in cost savings. In addition, the carrier may aggregate multiple customers onto shared over-provisioned access transport facilities. Packet technologies are also better adapted to real-time service provisioning. The carrier can realize savings from packet aggregation technologies by: more efficiently filling access pipes, interfaces and network devices; more efficiently utilizing switch interfaces and matrix capacity; and grooming smaller interfaces into larger, more cost effective interfaces. These technologies will be used in areas where high data throughput is required, and metro/wide area network integration and strict quality of service (QoS) are not main concerns. Packet-based technologies are optimized to support the following: network traffic that is primarily data, packetized video or voice; where delay sensitive applications can operate with less stringent QoS; where network migration and simplicity is needed; where bandwidth and installation costs are a concern; and where it is important for the carrier to leverage an installed base of Ethernet knowledge for network management, monitoring and trouble-shooting. Henderson (2001) notes that service providers can improve cost structures by aggregating presentation, delivery and support for IP-based communications services via communications portals. Communications portal providers are an emerging breed of IP enabled carriers who are providing net (Internet) telephony, unified messaging, and collaboration (i.e. application sharing, web-casting, voice chat) services. Rosenbush and Einhorn (2000) describe voice-on-the-Net as a growing international business. Companies around the globe are beginning to use new systems based on Internet technology in place of their old phone networks -- not only because they are cheaper but also because they can do so much more. Internet technology allows many calls to share the same phone line. Voice-on-the-Net is expected to account for more than $30 \%$ of total global telecom traffic by the year 2005 . As call quality via IP approaches or exceeds traditional circuit-switched calls, carriers will increasingly recognize the economic advantage to routing calls via the Internet (Dukart, 2001). The British-based global carrier, Cable \& Wireless, recently announced a major initiative to build a national state-of-the-art optical fiber network in Japan. This advanced network will enable the carrier to deliver a new standard in IP and data services to business customers throughout Japan (Stober, 2000).

\section{Optical Capabilities in the Access/Metro and Regional Networks}

Global communications carriers are also keenly interested in and pursuing optical networking capabilities such as: high speed transmission; dense wavelength division multiplexing (DWDM); optical layer management; and optical packet switching. In addition, they are working with vendors to develop next generation fiber, ultra broadband and optical cross connect systems, and more efficient wavelength amplifier systems. Much of this work is being driven by carrier challenges and objectives, such as the following:

- A significant increase in capacity demand in the near future (driven by data growth);

- A need for automated service provisioning capabilities;

- Requirements for higher spectral density to increase capacity on a per fiber basis;

- An on-going need to reduce cost, power consumption and physical size of future generations of transmission, transport and access equipment; and

- Carrier requirements for scalability, reliability and flexibility in the transport network.

Optical capabilities and WDM have traditionally been used in the regional and core or backbone networks. As capacity requirements increase and economics improve, these technologies are moving into the metropolitan transport network, and in some special cases, into the access network. Some of the larger ISPs, ILEC central offices and carrier hotel transport customers in major metropolitan cities may warrant WDM directly to their buildings to transport data at OC-48 or OC-192 rates. The main drivers for metro and access network optical capabilities include:

- Relief of fiber exhaust in metro areas via WDM;

- Better utilization of fiber facilities through packet technologies and statistical multiplexing; 
- Need to deploy next generation fiber that maximizes wavelength counts, improves performance, and minimizes signal dispersion and loss;

- Use of wavelength switching and optical cross connects for wavelength management;

- Need to deploy more efficient amplifiers to extend optical signals and lower costs; and

- Requirement to offer convergent services over efficient optical transport systems and networks.

According to Wolter (2000), a new class of products called optical access or WAN platforms, is bringing multi-service capabilities to metropolitan optical networks. These products aggregate a variety of inputs to an optical network -- IP, ATM, Ethernet, TDM-onto SONET rings or DWDM wavelengths. They can extend access to optical networks as far out as an office building, or can groom metro rings into large multi-protocol streams ready for backbone networks (Wolter, 2000). Li (2000) talks about Carrier1, a European-based global supplier of long distance communications services. Carrier1 is deploying a new fiber that increases the distance an optical signal can travel without dispersion compensation. Underground ducts are being constructed with this new fiber technology in Amsterdam; Carrier1 plans to build in 20 other major European cities, including Paris, Berlin and Zurich. The customer is demanding service flexibility, speed of provisioning, low cost per bit, and low cost per connection. WDM and other optical technologies are proving to be essential tools in the metropolitan market to meet these evolving needs.

\section{Summary and Conclusions}

There are many factors influencing the global communications carrier's network planning decisions and strategy. There are market place factors such as Internet growth, new IP services, voice and data convergence, non-traditional competitors, and global expansion. There are technology factors such as high speed switching, the proliferation of new access technologies, developments in optical technologies, declining costs, and new routing/control protocols. There are regulatory factors such as global deregulation and price arbitraging in traditional telecom services (i.e. VoIP by-pass and circuit-switched voice using ILEC switching). And there are business factors such as declining profit margins, the need for new services and revenue growth -- and the need to reduce access costs, operations costs and service intervals. All or some of these factors will be part of the global carrier's strategic decision-making process. Having frameworks and methodologies to objectively identify and assess critical factors, and consider alternative scenarios, becomes vital to the carrier. The carrier can learn valuable lessons from analyzing and studying specific business cases. The combination of past experiences from seasoned industry personnel, lessons learned from studies and analyses based on real experiences, sound business sense, clear corporate vision, and flexible objectives - will ensure the global communications carrier's success as it moves forward in planning and implementing new networks.

\section{References}

Albright, P. (2001, January). An Innovator's Dream Is another's Disruptive Technology. Wireless Week,1, $18-19$.

Dobrushin, A. (2000, December). Convergence Revisited: Will Intersection of Optics, IP Finally Realize

Promise of a Single Network. X-CHANGE,1, 32-36.

Dukart, J. (2001, January). IP, Latin America Emerge as Major Growth Markets. Phone+,1, 58-59.

Henderson, K. (2001, January). Service on a Portal: Web-User Interface Enables Enhanced Communications. Phone+,1, $36-42$. Li., J. (2000, June). DWDM Moves into the Metro. Outside Plant, 1, 46-51.

Martin, S., Deskey, B., and Pihl, A. (1999, January). 5th Annual Telecommunications Customer Expectations Survey Report. Deloitte Consulting LLC, $1,1-16$.

Rosenbush, S. and Einhorn, B. (2000, May). The Talking Internet: Special Report Telecommunications. Business Week,1, 174196.

Seals, T. (2001, January). Bundled Services: No Sign of Dwindling in 2001. Phone+,1, 52.

Stober, S. (Ed). (2000, December). News: Cable \& Wireless Creates Optical Fiber Network in Japan. Outside Plant,1, 16.

Strouse, K. (2001, January). Planning, Measuring, Managing Market Share. Phone+, 1, 94-96.

Summerill, S. (2000, December). Next-Gen Networks: Got Features?. Sounding Board,1, 38-45.

Wede, D. (2000, December). A Business Case for Fiber-to-the-Home. Optical Solutions, Inc., 1, 1-16.

Wolter, C. (2000, June). Optical Access Rides the Waves. X-CHANGE, 1, 8-14.

Wolter, C. (2000, July). Mighty Mice: Fledging Companies Make Waves at Optical Fest. X-CHANGE,1, $26-32$.

Wolter, C. (2000, December). Making Next-Gen Friends: VoDSL Vendors, Softswitch Makers Partner Up for the New Networks. Sounding Board, $1,46-50$. 


\section{Appendix A \\ Glossary of Technical Acronyms}

\begin{tabular}{|c|c|}
\hline ADM: & add drop multiplexer \\
\hline ASP: & alternative service provider \\
\hline ATM: & asynchronous transfer mode \\
\hline CAP: & competitive access provider \\
\hline CLEC: & competitive local exchange provider \\
\hline CPE: & customer premise equipment \\
\hline DAL: & direct access line \\
\hline DSL: & digital subscriber line \\
\hline DSLAM: & digital subscriber line access module \\
\hline DWDM: & dense wave division multiplexing \\
\hline DXC: & digital cross-connect \\
\hline E-n: & ETSI standard for digital carrier \\
\hline FITL: & fiber in the loop \\
\hline FR: & frame relay \\
\hline FWA: & fixed wireless access \\
\hline GbE: & gigabit Ethernet \\
\hline GDP: & gross domestic product \\
\hline HFC: & hybrid fiber coaxial cable \\
\hline IAD: & intelligent access device \\
\hline ILEC: & independent local exchange carrier \\
\hline IP: & Internet protocol \\
\hline ISDN: & integrated services digital network \\
\hline ISP: & Internet service provider \\
\hline IT: & information technology \\
\hline LAN: & local area network \\
\hline LD: & long distance \\
\hline LTE: & line termination equipment \\
\hline MDU: & multi-dwelling unit \\
\hline MUX: & multiplexer \\
\hline OC-n: & ANSI standard optical carrier \\
\hline OLT: & optical line termination \\
\hline PBX: & private branch exchange \\
\hline PCS: & personal communications services \\
\hline PL: & private line \\
\hline PON: & passive optical network \\
\hline POP: & point of presence \\
\hline POTS: & plain old telephone services \\
\hline PSTN: & public switched telephone network \\
\hline PTMP: & point to multi-point \\
\hline PTP: & point to point \\
\hline PTT: & postal telephone \& telegraph \\
\hline QoS: & quality of service \\
\hline SDH: & synchronous digital hierarchy \\
\hline SME: & small and medium enterprise \\
\hline SOHO: & small office/home office \\
\hline SONET: & synchronous optical network \\
\hline STM-n: & ETSI standard optical carrier \\
\hline TDM: & time division multiplexing \\
\hline VoIP: & voice over the Internet \\
\hline VSAT: & very small aperture terminal \\
\hline WAN: & wide area network \\
\hline WDM: & wave division multiplexing \\
\hline
\end{tabular}

